\title{
Profesiones emergentes y herramientas modernas de gestión de las organizaciones
}

\author{
Pierre Tripier*
}

\section{Introducción}

Aunque no pueda ofrecer datos estadísticos sobre la mutación de la cual voy a hablar en este trabajo, puedo afirmar que, tanto en América Latina como en Europa, la organización de las industrias y de los servicios ha estado sufriendo un cambio mayúsculo que consiste esencialmente en un proceso de sustitución dentro de las esferas directivas de las organizaciones. Hasta hace poco en esas esferas reinaban los responsables por jerarquía y por funcionamiento, y, actualmente, han estado siendo sustituidos por otra figura organiza cional: la del responsable/ consultor. ${ }^{1}$ La conclusión puede expresarse de otra manera: en la década de los cincuenta, cuando Dalton escribía en la American Sociological Review, los consultores en recursos humanos 0 en desarrollo organizacional estaban dentro de las empresas y pertenecían al círculo de responsables de las mismas; hoy

\footnotetext{
* Profesor de Sociología,Université de Versailles Saint Quentin en Yvelines, Vicepresidente del Institut de Rechereche et de Développement de la Qualité, Besançon, Francia. Se le puede enviar correspondencia a Laboratoire Printemps 47 Bd. Vauban 78047 Guyancourt Francia. Tel:33139255656, fax 33139255655.

Correo electrónico:pierre.tripier@ printemps.UVSQ.fr

1 Para llegar a esta conclusión me apoyo en un buen número de informes monográficos que conozco gracias a la red europea y latinoamericana a la cual pertenezco Red Memises, programa Alfa,Comunidad Europea, DG1.
} 
en día, no pertenecen a la empresa o se ven ayudados y apoyados por peritos externos que les aconsejan la introducción de cambios para que la empresa siga sobreviviendo (Dalton, 1950).

Otro resultado empírico que se observa en numerosas monografías recientes es la multiplicación de nuevos esquemas organiza tivos, que aparecen simultáneamente en el mercado mundial y reemplazan los que funcionaban hace algunos cinco o diez años. Y si no los reemplazan, al menos se introducen al lado de los que ya existían sin que nadie se preocupe de los primeros y sin ver que algunos de los procesos nuevos no son compatibles con los anteriores (Cuq, et.al, 1998).

Estos dos resultados empíricos han conducido a nuestra red académica a estudiar las características de las nuevas formas de organización que han introducido los consultores con la aprobación de los responsables de las empresas. De ahí que hayamos concentrado nuestras investigaciones en: la calidad total, en el manejo de proyectos 0 ingeniería paralela, en los balances de competencias y en la reingeniería. Ciertamente esta última - la reingeniería- se encuentra poco desarrollada en los países europeos en los cuales viven los miembros de nuestra red (Bélgica, Portugal y Francia).

\section{Morfología de las herramientas modernas de gestión}

Si alguien examinara los rasgos característicos de las herramientas de gestión de las organizaciones que hemos estudiado, $s e$ daría cuenta que todas tienen formas similares. Sin entrar en el carácter peculiar de cada una, se podría decir que tienen tres rasgos sobresalientes: a) su carácter completo y auto-adaptativo, b) el estar centradas en el propósito de resolver problemas y la necesidad de dar una forma especial a los datos de manera que permitan lograr soluciones; y c) el hecho de poseer recursos micro-políticos que les permiten entrar a las empresas, imponerse y extender su influencia. 
Carácter completo y autoadaptativo

Para entender lo que queremos decir por completo y auto-adaptativo, tomemos el ejemplo de la herramienta de gestión organizacional más antigua que hayamos estudiado, esto es la herramienta lla mada calidad. El desarrollo de esta herramienta nos permite entender cómo, poco a poco, se pasa de un instrumento sencillo, con solo un objetivo, a algo complejo y eventualmente sistémico.

Si seguimos paso a paso la historia de la herramienta llamada "ca lidad", vemos que al principio se desarrolló junto con la estadística industrial, aparejada a la convicción de que si se pudiera disminuir la desviación estándar de la producción de cada obrero en relación a cada máquina, entonces aumentaría la calidad de los productos ( $\mathrm{Ba}$ yart, 1995).

En esta primera fase, ya aparecen algunas expresiones del principio que llamo "el principio de la igualdad cognoscitiva". En efecto, los primeros estadísticos que introdujeron la idea de calidad usa ban una ficha para cada obrero, quien registraba las medidas de fa bricación sobre una muestra de su propia producción, sin que na die los controlara. Así se hacía, con la seguridad - de parte de los que introdujeron estas técnicas- de que los obreros son los primeros en saber de dónde provienen los resultados defectuosos.

Esto significa que para descubrir el origen de un defecto se necesita la cooperación entre ingenieros y obreros, y para lograr esta colaboración, cada uno debe respetar el saber del otro: el entendimiento pragmático, local y práctico de los obreros, así como el sa ber abstracto, general y matemático de los ingenieros. La igualdad cognoscitiva no significa que uno puede ocupar el papel del otro, sino que cuando hay un problema que resolver, sólo la colaboración de ambos permite su resolución: el ingeniero puede entonces aprender del obrero y viceversa.

Pasado el tiempo, cuando Deming y Juran (ambos ayudantes del estadístico Shewhart que impuso en la Compañía Bell las primeras acciones de calidad) van al Japón, el concepto se enriquece cuando los japoneses les enseñan que más vale pensar que el origen de los defectos es sistémico, en vez de imaginar que pueden ser el simple resultado de los errores de un solo responsable. 
A su vez, Deming - que llegó al Japón acompañando a Mac Arthur para llevar a cabo el censo de este país arruinado por la guerra y el bloqueo naval- discutió con el presidente de la jusE (Ishikawa) y le insistió sobre el carácter tan frecuente de la proporción descrita por Pareto en relación a la repartición del producto nacional, esto es, según Pareto el $\mathbf{8 0} \%$ de las fortunas pertenece al $20 \%$ de las familias. De esta proporción se deduce que, en vez de tratar de obtener partes del mercado del $\mathbf{8 0} \%$ de los habitantes más pobres del planeta, sería conveniente que los japoneses conquistaran el mercado del $20 \%$ más rico. Esta recomendación implicaba que los japoneses no gastasen el tiempo intentando reanudar la política industrial y comercial que tenían antes de la guerra, cuando Japón inundaba el mundo entero con productos baratos y de muy escasa calidad, sino que habría que vender a los más ricos. Se les debían ofrecer las mercancías a las cuales estaban acostumbrados, pero de mejor calidad y más baratas. Además, tenían que informarse sobre los deseos poco explícitos de los consumidores de países ricos para sa tiffacer necesidades todavía desconocidas.

Después de hacer su trabajo estadístico, el de detección de errores y el de estrategias industriales y comerciales, los inventores de la idea de calidad vuelven a los Estados U nidos cuyas industrias automotrices pierden partes importantes del mercado mundial que ha bían sido conquistadas por los automóviles europeos (sobre todo alemanes y suecos) 0 japoneses. Fue entonces cuando Deming fue reclutado como consejero del presidente de la Compañía Ford y decidió hacer todo lo posible para reducir las desviaciones estándar en cada acto productivo. Al mismo tiempo, impuso que los obreros y los empleados fueran escuchados cada vez que detectaban un defecto productivo y organizacional. En vez de tener un verdadero ejército de inspectores que verificaran el trabajo de la base, impuso que se tuviera confianza en los que laboraban en el nivel más básico de la organización. Igualmente, introdujo sus famosas fichas de auto-comprobación que se usaban desde hacía ya unos cuarenta años.

Los resultados permitieron mejorar la situación pero no se llegó al nivel de excelencia deseado. La leyenda dorada en la que baso este relato (Gabor, 1992), pretende mostrar que Deming se dio entonces cuenta de que el hecho de que la reducción de las des- 
viaciones estándar no haya sido respetada, provenía de los provee dores de Ford, porque ellos no aplicaban sus métodos. En ese momento, le vendría la idea de aplicar un sistema de certificación de productos, pero también de procesos de producción (es decir de organización y de tecnología) ya utilizados por la NASA y la industria nuclear. Así, expandió la certificación en toda la industria automotriz, desde el más grande hasta el más pequeño abastecedor. De esta manera, nació la necesidad de tener normas mundiales, como las llamadas Is0 9000. La primera línea de estas normas, establecía que para certificar una empresa debía tener un responsable de la calidad y un lugar, conocido de toda la empresa, donde estuviera ubicado.

Deming igualmente añadió que no solamente había que fijar reglas respetadas en la empresa y por los proveedores, sino que se debía también, de manera periódica,erradicar los errores técnico-organizativos descubriendo de dónde provenían. Para esto se debía suponer, como lo indicaba Ishikawa, que un defecto percibido tiene una causa inmediata que se puede descubrir con algunos sencillos indicadores y siguiendo un modelo mecanicista simple. Sin embargo, hay que reconocer que la causa de un defecto tiene, a su vez, una causa más profunda que la que surge de la concepción errónea de que todo se explica por la tecnología utilizada (Ishikwa, 1984).

Con estos ejemplos, se puede ver cómo la vida propia de una herramienta de gestión - la calidad- hace que, poco a poco, pase de ser una simple función técnica y se convierta en un conjunto de opciones cognitivas y de soluciones micro-políticas, acompañada de la constitución, dentro de las empresas, de un verdadero poder de influencia al servicio de los ejecutivos. ${ }^{2}$ Como se observa, los tres niveles de Platón están contenidos dentro del mismo dispositivo organizacional, pero se añadió uno que viene a ser más bien de origen aristotélico: la capacidad de auto-corrección permanente mediante los actos incesantes de mejoramiento de la calidad.

2 No conozco toda la literatura latinoamericana sobre la calidad, ni siquiera toda la mexicana, pero dentro de nuestra red, el libro favorito es Calidad Total de Stolovich, Lescano y Morales (1995), Montevideo, CIDEUR/ FESUR. 


\section{Un modelo: el robot de tercera generación}

Si uno buscara una metonimia para entender mejor la forma que adquieren las herramientas modernas de gestión, me parece que se podría utilizar la del robot de tercera generación, porque éste es ca paz, una vez que se le ha dado una linea de acción, de adaptarse al terreno donde debe trabajar $y$, al mismo tiempo, es bastante completo y autosuficiente para poder permanecer autónomo. 0 tro rasgo esencial de los robots es el siguiente: están alimentados por datos ya precodificados, si no fueran así, entonces los datos no tendrían ninguna significación.

Veamos el valor de la metonimia. La acreditación de competencias, así como la calidad total, el control de proyectos (o ingeniería paralela) y la reingeniería, ciertamente pasan por algunos momentos de creatividad sin formalización, pero siempre arriban a un momento en el cual las informaciones han de tomar una forma muy estricta para poder entrar en la computadora, la cual va a crear la memoria del pasado, pero también ayudar en la realización del porvenir. La gran diferencia entre estos instrumentos y el robot radica en que este último es caro y cuando una empresa lo compra es que quiere realmente utilizarlo. Además, debe de estar ya preparada pa ra hacerlo, de modo que al introducir nuevos robots, la empresa es té lista para reemplazar, por ejemplo, todo un taller.

Esta consecuencia no necesariamente ocurre con las tecnologías blandas que son herramientas de gestión, las cuales pueden ser utilizadas por un simple mimetismo estratégico: una rama, una empresa, utiliza una herramienta de gestión, si ésta ya es conocida por otras empresas y $\mathbf{s}$, al aplicarla, suponen que se puede desequilibrar el mercado 0 arrebatarles partes del mercado.

El mimetismo estratégico aparece también con el uso de máquinas 0 de políticas financieras; sin embargo, como las herramientas de gestión parecen constreñir menos a las organizaciones, ya que se aplican a seres humanos que se suponen son seres adaptables, entonces, pocas personas piensan que las herramientas de gestión pueden dejar huellas perdurables. Por eso, en la mayoría de las ocasiones las organizaciones guardan viejas maneras de hacer las cosas que suelen ser incompatibles con las nuevas formas de organización. 


\section{Las herramientas de gestión son completas y cerradas porque integran recursos micro-políticos}

Es por las razones anteriormente descritas que los instrumentos de gestión terminan por tener, en su propia composición, recursos micro-políticos. Como son diferentes maneras de transformar las organizaciones y luchar contra la irreversibilidad que nace de la existencia irremediable de las rutinas y las tradiciones, y como para realizar los cambios, intentan llevar a la práctica la igualdad cognoscitiva y unir a todos miembros de la organización en las actividades de solución de problemas, entonces, por consecuencia, terminan por imponerse. Esta imposición de los cambios emanados del uso de instrumentos de gestión, no proviene del hecho de que son en sí mismos racionales - sabemos que dentro de las empresas y las organizaciones se cruzan múltiples racionalidades adversarias- , sino de que las mismas herramientas ofrecen, a quienes las usan, los recursos micro-políticos que les permiten introducir los cambios.

Aquí también debo regresar al tema de la calidad, ya que esta herramienta, junto con las normas Iso 9000, trata de difundir por el mundo entero su forma mínima de organización. Cuando se analizan estas normas uno puede ver cuáles son los recursos micro-políticos a su disposición:

\section{Recursos organizacionales}

El primer grupo de recursos es de tipo organizacional; es decir, los que permiten a los que introducen la calidad dentro de las empresas, existir e imponerse y les dan un lugar y un puesto con responsabilidades específicas, una dirección específica también, una meta, además de la certificación y los procedimientos para obtener dicha certificación.

El responsable de la calidad debe producir un manual de calidad. Para establecer este manual debe solicitar la ayuda de cada miembro de la empresa y escribir o hacer escribir la rutina del trabajo de ca da uno. De esta manera puede entrar en contacto con los detalles 
más insignificantes y minúsculos de la organización, por lo que se explica que el responsable de la calidad llega a tener, a la vez, una visión detallada y global de toda la empresa.

\section{Recursos persuasivos}

El responsable de la calidad debe medir y contar; es por ello que le corresponde determinar, por ejemplo, cuáles son los costos que tienen todas las acciones que visiblemente se hacen sin-calidad, así como de las mercancías que le regresan al fabricante por defectuosas. Además, mide otros costos más difíciles de precisar como son aquéllos que provienen de la existencia de servicios internos inútiles. Las bitácoras que surgen de esta contabilidad, le sirven al responsable de la calidad para persuadir a otros responsables cuyas metas son diferentes: por ejemplo, un director de producción tiene por meta fabricar una cantidad predeterminada en un tiempo límite establecido. Para él, permitir que sus obreros sigan cursoso atiendan reuniones sobre la calidad es totalmente contrario a sus objetivos. Pero si el responsable de la calidad demuestra que los costos de la no-calidad son mucho más elevados que los del tiempo perdido pa ra entrenar a los obreros, entonces puede ganarle la partida.

\section{Recursos para reclutar aliados}

Las normas iso 9000 prevén que al menos cada año se evalúen los resultados de las acciones de mejoramiento de la calidad y también suponen que hay en cada empresa u organización un comité de control de la calidad, compuesto por todos los directivos importantes. Estas son ocasiones para sensibilizar a los miembros más influyentes y al director general sobre las metas del servicio de la calidad.

Sin describir con mayor detalle estos dispositivos, quisiera subrayar el hecho de que cada herramienta tiene, sin duda alguna, los recursos que permiten imponerse a los demás. Es por eso que estas herramientas nos parecen cerradas sobre sí mismas y, a la vez, son completas;'es decir, juntan, con cierto nivel de coherencia, elementos de orden cognitivo con otros de orden técnico y micro- 
político. Esto es, establecen cómo se deben utilizar y, al mismo tiempo, quién debe hacerlo.

La prueba de que los recursos micro-políticos cierran las herra mientas de gestión es un hecho irrefutable: los procesos de ingeniería paralela fallan una de cada dos veces. La ingeniería paralela es interesante de estudiar, ya que reúne gentes de disciplinas científicas diversas y secciones de la empresa diferentes para trabajar juntas en un mismo proyecto. Para realizarlo, deben sacarlo del contexto factual y pensarlo en un mundo abstracto, de tipo newtoniano y, después, cuando el proyecto ya está listo, lo ponen de nuevo en su contexto real. Es en ese momento en el que, las mayoría de las veces, el proyecto fracasa porque la ingeniería paralela ha sido pensada como un proceso y no como una herramienta de gestión. Es por eso que es tan crítico el momento en el que el proyecto debe tomar en cuenta las rutinas y tradiciones de la empresa.

Estas evidencias conducen a la hipótesis que anuncié al principio: la morfología de las herramientas modernas de gestión organizacional termina por multiplicar a los consultores, ya que, a pesar de que se cuenta con numerosas indicaciones explícitas en las conferencias y en los libros que presentan las nuevas herramientas, los responsables insisten en que esto nunca es suficiente. Lo anterior significa que para los responsables de las organizaciones siempre hay cierto conocimiento que se presenta en estado esotérico (por ejemplo: cómo aplicar las normas, cómo persuadir a los otros, cómo impedir que seis meses después de introducir una herramienta su utilización se desvíe, etcétera). Este saber oculto se supone que está en manos de un consultor.

A su vez, la empresa introduce, a menudo, las nuevas herra mientas de gestión en un acto de mimetismo estratégico. Esta ma nera de comportarse produce lo que llamamos en Francia el zapping de management. Para llegar a tener una ventaja estratégica sobre sus competidores (internos o externos), cada responsable quiere introducir un instrumento que no haya sido utilizado por los otros. Viendo esto, los otros lo copian, y así se va la cadena interminablemente. Esto es posible por que cada herramienta tiene dispositivos técnicos, pedagógicos y micro-políticos, como lo describía anteriormente. 


\section{Fisiología de las herramientas modernas de gestión}

Hemos visto hasta ahora cuáles son los rasgos principales de la morfología de los instrumentos de gestión. Veamos cuál es el contenido que los hace ser tan modernos. La tesis que sostiene nuestra red euro-latinoamericana es que el contenido (la fisiología) de estos instrumentos está dada por dos rasgos que ya he apuntado: primera mente, son herramientas de naturaleza cognoscitiva, es decir, sirven para plantear y resolver problemas; en segundo lugar, son instrumentos que crean una tensión, quizás inédita, entre las teorías igualitaristas y desigualitaristas de nuestra civilización.

Las herramientas modernas de gestión son a la organización lo que las nuevas normas y las nuevas máquinas a la fabricación: crean una nueva definición del trabajo

En el siglo xVIII el trabajo era, a la vez, un intercambio y un fenómeno físico. Por ejemplo, en la gran enciclopedia de Diderot y d'Alembert se subrayaba el aspecto de intercambio: "Trabajo:0 cupación cotidiana a la cual se ve condenado cada ser humano por su menester y a la cual cada uno debe al mismo tiempo su salud, su serenidad, su sensatez y a lo mejor su virtud". Un poco más tarde va a establecerse la ley de la conservación de la energía según la cual su característica fundamental es que no puede ser creada ni destruida, pero ś transformada. El concepto de trabajo era equivalente al impacto de una energía sobre un punto. De hecho, las medidas de energía y las de trabajo eran equivalentes [ $1 \mathrm{erg}$-metro newton = 1 joule].

Se utiliza el concepto de trabajo para pensar en dos tipos de intercambio equilibrado: la equivalencia, por un lado, entre la energía usada por una persona y, por otro, lo que recibe como rédito, interés en el trabajo, realización de sí misma y equilibrio psicológico; esto también se aplica a las sociedades: la equivalencia entre la energía gastada por una nación y su nivel de enriquecimiento. Es en la ciencia física en donde este concepto se crea y en donde todo está dirigido a establecer precisamente una equivalencia.

Organizadas de manera subyacente por este modelo o paradigma, las sociologías del trabajo han tratado de entender cómo nace una acción en el contexto de una situación, contestando la si- 
guientes preguntas: ¿cuánta energía ha necesitado este nacimiento?, ¿quién la ha abastecido?, ¿cómo la equivalencia entre diferentes gastos de energía se ha equilibrado?

Cuando leemos a Babbage, siguiendo la invitación de J. J. CastiIlo (1994), o cuando revisamos las recomendaciones de Weber pa ra hacer los análisis psicofísicos sobre el trabajo, o cuando $s$ lee a Marx, Taylor, Naville, Touraine, Walker y Guest, o a Clark Kerr, esta mos en este mundo completamente sumergidos dentro del problema de las equivalencias. Para Marx, por ejemplo, la equivalencia es una necesidad intelectual, política y moral: hay que entender, según él, por qué las promesas de los liberales de construir, con la democracia, una sociedad igualitaria ha fallado y el centro de la explica ción termina por ser la explotación capitalista; es decir, el hecho de que se le obliga al asalariado a regalar al capitalista horas de trabajo gratis. Para Weber, es una necesidad factual y científica: estamos en un mundo en el cual el capital disponible es escaso porque depende de su contrapartida en oro o plata: "Las transformaciones tecnológicas, a consecuencia de la escasez de 'capital' disponible, siguen el camino que indique la obtención del máximo ahorro en los cos tos" (Weber, 1908/ 1994:31).

Los protestantes para quienes, como Taylor, Veblen o Deming, la eficiencia productiva es un deber moral muy por encima que cualquier otra cosa, los cambios técnicos u organizativos que llevan a una mayor productividad son vistos como un momento normal del desarrollo evolutivo. La destrucción productiva puede producir penas y llantos, pero como imperativo moral permite al mundo enriquecerse sin trabajar inútilmente Como se puede apreciar, toda esta manera de ver está enteramente contenida en una física de la equivalencia de las energías.

La insistencia de concebir al trabajo como solución de problemas es tal vez producto de los ćŕrculos de calidad en donde los métodos de resolución de problemas se utilizan casi de manera sistemática. Lo que me parece importante resaltar aquí es que esta visión del tra bajo juega como un paradigma "kuhniano"; es decir, arregla de ma nera distinta los conocimientos anteriores y, sobre todo, no insiste más sobre $\mathbf{E}$ aspecto tan polémico de la equivalencia entre energía 
gastada y enriquecimiento. De este modo introduce, dentro de una problemática física, nociones más "blandas" tomadas de las ciencias sociales y las humanidades, mitigando, a su vez, la explotación del hombre por el hombre y exaltando la capacidad individual de plantear y resolver un problema. Los trabajos de Newell y Simon (1963 y 1972) muestran que, ante una situación inédita, la capacidad de lograr un objetivo depende de la capacidad de cada uno de tener una idea clara del problema que se debe solucionar.

Cuando una cuestión es compleja, cada uno utiliza una epistemología simelliana: fija los límites temporales de la acción, utiliza la información que ya tiene a la mano producto de situaciones anteriores y, sobre todo, compara la forma del problema con situaciones que ya le son conocidas; utiliza soluciones que han funcionado en otros lugares, ante otros dilemas, pero cuya utilización ya se conoce bien, en donde se sabe cómo dominar la situación utilizando dichas soluciones.

Esta manera de ver las cosas en la industria permite a la vez leer con otros ojos algunos trabajos clásicos de la sociología del trabajo y de la organización; si insistimos sobre los problemas del conocimiento, abren la puerta a la comunicación con los tecnólogos, los psicólogos y los etnólogos del trabajo, y con los estudiosos del ma nagement y de la lingüística; posiblemente, cierra también algunas comunicaciones que se tenían con la historia, las ciencias políticas y la economía. Es lo que trataré de mostrar en el siguiente apartado.

\section{Otras comunicaciones}

Leer de nuevo a los clásicos

Uno de los autores menos ortodoxos de la sociología del trabajo es seguramente D. Roy, cuya experiencia como obrero pudo demostrar que los obreros, cuando no siguen las órdenes de sus superiores sino sus propios conocimientos acerca de una situación, pueden resolver los problemas mejor que siguiendo las órdenes de sus capataces y otros jefes Roy demostró también que el hecho de que los traba- 
jadores controlen su propio trabajo puede ser funcional a los objetivos de los dueños de una empresa (Roy, 1954).

Los obreros de quienes hablaba Roy en esa época, tenían que desobedecer las órdenes de sus superiores porque éstas surgían de una visión abstracta, digamos general, acerca de la manera en la que se debe manejar una planta productiva. Estas suelen ser las visiones que saben enseñar los consultores y los ingenieros, pero que tienen poco que ver con la vida laboral local.

La cuestión anterior fue discutida en Francia por J. D. Reynaud en los años ochenta. Este autor demostró que en las empresas existían dos tipos de conocimientos, uno singular, local - el de los trabaja dores- que permite resolver problemas particulares, y el otro - el de losingenieros- que permite extender este conocimiento, encontrar las raíces generales de los defectos (Reynaud, 1988). El primero permite reivindicar una "regulación autónoma" de la situación de trabajo, contra la "regulación coactiva" de los ingenieros. Reynaud propone que entendamos la vida organizativa como producida por la "regulación conjunta", compuesta de las dos formas de regulación.

Si se toma el trabajo como una manera de resolver problemas, este carácter complementario propuesto por Reynaud parece aceptable ya que sin la regulación autónoma no se puede plantear correctamente el problema y sin la regulación coactiva no se puede solucionar definitivamente.

Las herramientas y los procesos técnicos son métodos fijos de solución de problemas

Cuando se dibuja un objeto técnico (Gay, 1994; Coiffet, 1994), como cuando se concibe una herramienta o un proceso técnico, uno aplica sistemáticamente las diferentes fases de un proceso de conocimiento aplicado. Para hacerlo se deben contestar las siguientes preguntas:

- ¿Qué? (a qué necesidad corresponde).

- ¿Cómo? (¿se van a poder cumplir las necesidades funcionales y al mismo tiempo corresponder a las costumbres culturales de manejo y utilización?). 
- ¿Por qué esta herramienta y no otra? (aquí se la compara con soluciones alternativas, los logros de los competidores, con capacidades económicas y técnicas de realización).

- ¿Con quién? ¿cómo va a ser conectada con otras herramientas, otros procesos? (Aquí la historia de las máquinas, como la que documentó el INIDET ${ }^{3}$ es de una gran importancia para responder a la pregunta).

Si aceptamos que trabajar es también resolver problemas, entonces nosotros los académicos podemos hablar con los tecnólogos, puesto que nuestros instrumentos de comprensión son los mismos.

Las pautas, las normas y los procedimientos

son también maneras de resolver problemas

Las normas también existen para contestar a la pregunta: ¿cómo debemos hacer las cosas? Éstas definen el problema (qué), de qué manera hay que manejarlo (cómo), por qué hay que utilizar tal regla particular (por qué), y cómo esta pauta se combina con otras ya existentes (con quién).

Si comparamos las máquinas y otras herramientas con las normas, observaremos que estas últimas tienen dos particularidades.

1a. Pueden resolver problemas para una persona y crear problemas para otras. Como su modo de construcción es a menudo menos dialógico que el de las maquinarias y como son utilizadas en ámbitos de relaciones desiguales, uno nunca puede estar seguro de su capacidad real de generar soluciones.

2a. Pueden ser interpretadas de distintas maneras ( $y$ hasta olvidadas) ya que su carácter flexible proviene del hecho de que se expresan y existen con el lenguaje y por el lenguaje. Recordemos que el lenguaje tiene un carácter dialógico, por tanto, ningún enunciado es perfectamente factual, ni perfectamente unívoco, salvo para

3 INIDET, Instituto Internacional de estudios sobre la Eficiencia Técnica, al cual pertenecen tres docentes que viven en México: Mónica Casalet de flacso; Rigas Arvanitis y Daniel Villavicencio de la UAM Xochimilco. 
quien lo pronuncia. Este fenómeno ha sido profundizado por el filósofo Paul Ricoeur para quien "la interpretación parte de la determinación múltiple de los śmbolos, pero cada interpretación reduce esta riqueza, esta multitud de sentido, y 'traduce' el símbolo según una clave de lectura que le es propia" (Vasilachis de Giordano, 1992:182).

Es justamente por eso que el lingüista Culioli piensa que "en el lenguaje común, entender al otro es un caso particular del equivoco, del malentendido". Es precisamente por esa razón que los positivistas lógicos prefieren un lenguaje lógico, que sea unívoco, 0 al menos lo parezca.

\section{Las herramientas modernas de gestión}

\section{organizan la tensión entre el carácter cognitivamente desigualitarista e igualitarista de nuestra cultura occidental}

El segundo punto sobresaliente de las herramientas modernas de gestión, es que tratan de resolver una de las mayores contradicciones de Occidente, la que surgió con el debate central del siglo XVI (entre protestantes y católicos).

La matriz anti-aristocrática creada en el siglo xvı por Calvino y desarrollada en el siglo siguiente por los pastores no-conformistas ingleses, se ubica al final de la época de losTudor, periodo en el cual el sistema feudal es desechado de las sociedades europeas. La desa parición de este orden social alimentó una nueva manera de ver las cosas, de suerte que resultaba intolerable la proporción de vagos, mendigos, desocupados y bandidos que caracterizaba a esas sociedades, al mismo tiempo, se desarrollaba una burguesía que quería ser completamente independiente del estado y de la iglesia. Esta matriz reposa sobre cinco argumentos:

1. La igualdad de todos los individuos dentro de la comunidad.

2. La igualdad es necesaria para que cada uno siga el camino que le indique su vocación. Esta vocación, que los padres de la iglesia 
concebían como una relación abstracta con Dios (en la cual la vocación más alta consistía en retirarse del mundo y vivir recluido en un convento), Calvino la redefinió intentando demostrar que en realidad era una vocación terrestre, la cual suponía que cada quien debía seguir el oficio al que se sentía llamado por su convicción personal. El mundo de espacios sociales en el que cada quien tenía que cumplir un papel que habría de repetir durante toda su vida - como lo veía Santo Tomás de Aquino- fue sustituido, por Calvino y sus discípulos, por una dinámica personal en la que cada uno, para honrar mejor a Dios, sigue el trayecto ocupacional al cual se siente atraído.

3. Para que cada quien pueda seguir su vocación personal, nadie debe estar sujeto a consideraciones secundarias. Por cierto, el matrimonio es una de éstas. Por eso los calvinistas consideraron que casarse no era un sacramento sino meramente un contrato entre dos personas, al grado que algunos de ellos, como Richard Steele (ver Miegge, 1989), pensaban que las vocaciones femeninas eran semejantes en importancia a las vocaciones masculinas.

4. Para que cada uno sea fiel a su vocación y que ésta se traduzca en la mayor gloria de Dios, cada ser humano debe ver su capacidad aumentada por la enseñanza - el hecho de aprender lo más posible- y también debe conservar su energía, su vitalidad mediante la higiene personal, el deporte y la actividad física.

5. Pero, sobre todo, cada uno debe invertir su energía en una actividad útil para la comunidad. El deporte lo es, ya que permite defender a la comunidad y mantener la energía vital de cada individuo. Estos valores se oponen a las fut lidades, al uso de servidumbre excesiva y al ocio de los nobles. La eficiencia productiva, el hecho de lograr hacer más cosas en menos tiempo, con menos esfuerzo, es un deber moral, ya que multiplica la utilidad de ca da uno dentro de la comunidad, al servicio de ésta como de la gloria de Dios. Ambas productividades son importantes, tanto la del trabajo industrioso como la del trabajo doméstico.

Esta matriz no solamente se halla escondida detrás del espíritu del capitalismo descrito por Weber y detrás de los ataques deVeblen contra la civilización de la clase ociosa sino también en los pisos 
subterráneos de la ley del valor de Marx y Engels. Inclusive las "ideas" recientemente presentadas bajo el rótulo de la posmodernidad que auguran el fin del trabajo industrial (Bridges, 1996) presentan este tipo ideal como el que va a reinar en el mundo entero; se dice que no habrá más ningún asalariado, cada uno será el dueño de su propio trabajo y podrá celebrar contratos con quien quiera y para lo que sepa hacer.

Esta matriz es también la tentación o la utopía de los instrumentos modernos de gestión. Como vamos a verlo más adelante, estos sirven para plantear y resolver problemas y parten del principio que trabajar es también plantear y resolver problemas. Es claro que para ciertos instrumentos de gestión organizacional, esta afirmación es adecuada; éste es el caso de la acreditación de competencias, en donde la cooperación entre los conocimientos locales y particulares, con los conocimientos generales y universales es necesaria. Pero en otras circunstancias la cosa no es tan sencilla. Esto es lo que explica el nacimiento de la reingeniería.

Ilustración de lo que precede

El hecho de suponer que cada hombre trata de abrirse un camino vocacional en donde hará las cosas lo mejor posible, o de suponer que las empresas van bien si logran saber cuáles son los deseos todavía no formulados de sus clientes (teoremas a los cuales se añade el de "trabajar es plantear y solucionar problemas"), pesa mucho en el posible éxito de las herramientas de gestión sobre las cuales hemos estado discutiendo hasta ahora.

Si pensamos en dos herramientas muy cercanas a nosotros como la reingeniería y la calidad total, veremos que ambas difieren en cuanto al papel quecumplen y por el hecho de que no tienen la misma mirada, ni la misma disciplina intelectual de referencia. La calidad total parte del deseo del cliente y construye su lógica de acción desde este punto de partida, respetando, paso a paso, la igualdad cognoscitiva. La reingeniería, por su cuenta, organiza su acción a partir de la estrategia de la dirección de la empresa y mide todas sus acciones desde este punto de referencia. 
La calidad total como un ensayo de conciliar las tensiones: igualdad cognoscitiva en la solución de problemas

Hemos visto anteriormente que la calidad total se presenta como un método que permite disminuir los defectos técnicos y organizativos de una empresa, y que se propone un meta inalcanzable: producir sin ningún defecto. Pero, ¿de qué defecto se trata? se puede tener en mente una organización perfecta, entonces todo lo que no corresponda a esa organización perfecta sería considerado como un defecto. Sin embargo, s esta falla es solamente estética, o si el único que se da cuenta de que hay un defecto es el jefe de la organización, entonces reducirlo sería un lujo inútil, un gasto innecesario de "sobrecalidad". El defecto tiene que ser un defecto para alguien, y ese alguien es, antes que nadie, el cliente. Ahora bien, esta concepción se ha extendido mucho y algunos consultores en calidad hablan también de defecto para el empleado, el cual se ve concebido como un cliente interno de la organización.

Por una parte, el empleado es un cliente interno y, por otra, es un actor para resolver los problemas. Los estudios que ha reunido nuestra red euro-latinoamericana $M$ emises muestran que esta manera de definir el papel del empleado es difícil por distintas razones. La más importante de ellas es quizá que las culturas son todavía jerárquicas y piramidales y es arduo pedirle a alguien que se exprese hoy como un obrero especializado sometido al control de los capa taces y mañana como miembro de un grupo igualitario en busca de la solución de problemas. En un estudio sobre las empresas que han obtenido en 1993 y 1994, los premios franceses de la calidad, esta dificultad era muy clara, ya sea porque las empresas no sabían reconocer los esfuerzos individuales, porque daban únicamente recompensas individuales donde claramente los avances hacia la calidad total eran producto del trabajo en equipo (Denioeul et al., 1995).

Es difícil introducir al cliente en la concepción organizacional cuando se trabaja con una retroalimentación lejana o incompleta

Nuestra red ha estudiado algunos casos en los cuales, aun si se sabe bien lo que quieren los clientes, sucede lo siguiente: cuando el 
producto está listo muchos de los clientes ya han desaparecido o han cambiado de opinión. Por ejemplo, para crear una nueva manzana se necesitan unos quince años de plazo, para abrir un pedazo de autopista son unos veinte años que deben transcurrir entre los primeros estudios y la inauguración. Se ve que en estos casos extremos, el acto de predecir los anhelos de los futuros clientes es extremadamente difícil.

Otro caso es el de la innovación técnica, donde no se sabe hasta el último momento cómo van a reaccionar los clientes. Hay casos como la PSl de la IBM en donde los estudios previos fueron buenísmos. Sucedió que los clientes declaraban que les interesaba mucho tenerlas y, curiosamente, poquísimos las compraron. Cuando se tra ta de decisiones a largo plazo o de mutaciones técnicas, las empresas deben tomar sus responsabilidades y decidir sin la ayuda de las informaciones provenientes de los clientes o considerando que éstos no necesariamente entran dentro del dispositivo de la toma de decisiones.

La reingeniería como un método para superar

los límites de la calidad total

La reingeniería no se ha desarrollado mucho en los países europeos a los cuales pertenecen los miembros de la red a la que pertenezco, así que no tenemos, como para la calidad total, una profundización histórica que nos permita juzgarla. Lo que han visto nuestros colegas latinoamericanos es lo siguiente:

Mejor visibilidad de la estrategia

Los límites de la calidad total pueden resultar de una dependencia demasiado fuerte de la opinión de los clientes (y todos los estudios sobre el consume's sbehavior muestran que esta opinión puede ser muy inestable) y pueden resultar también del hecho de tener que esperar un tiempo muy largo para recibir retroalimentación. Los límites pueden surgir también de intereses particulares que inva den partes de la empresa según el tipo de relación que cada una 
tiene con el entorno. 4 La reingeniería trata de evitar estos defectos, retomando la visión prometeana clásica según la cual el director de la empresa es el que toma las decisiones estratégicas y asume su pa pel retórico: cuando el director ha hablado, todos los demás saben lo que deben hacer y no hay objeción posible. De esta manera, la reingeniería permite medir la actividad con más eficacia que la ca lidad total. Los planes estratégicos se delegan al nivel de cada actor de la organización y la medida principal de cada actividad se define en función de la desviación respecto a metas establecidas y negocia das cada año.

\section{Medir las actividades en función de la estrategia}

La utilización del análisis funcional y la medida del valor permiten, en principio, orientar la estrategia de toda la organización y delegar a cada persona los objetivos con referencia a ese eje central. Aś, la herramienta llamada reingeniería, parece entonces un poco ambigua. Uno de sus inventores, James Champy (Champy, 1995) expone así el dilema de cada empresa: za quién debemos obedecer: al cliente 0 al accionista? Pero la respuesta es distinta a la que espera ríamos: hay que obedecer a los dos, y para hacerlo, hay que seguir la estrategia de los dirigentes. Así, un canto de amor hacia la libertad de cada uno - la calidad total- se transforma en los pocos ca sos que conocemos, en una oda a la gloria del management político en el más clásico estilo que podamos imaginar.

Difícil guardar la igualdad cognitiva, sin embargo, se pueden aumentar los recursos micro-políticos

Con los pocos datos que tenemos sobre empresas reingenieradas, diríamos que corresponden más a la cultura autoritaria clásica, no obstante, las empresas pasan por muchas dificultades siempre con el objetivo de que los métodos de solución de problemas sean utiliza-

4 Es este un tema clásico de la sociología de la organización planteado por Selznick en los años cuarenta, ver Selznick (1949). 
dos con eficacia.Ahora bien, hay que reconocer que, de hecho, en estos escasos puntos de verificación que poseo, las armas micropolíticas estuvieron bien utilizadas.

\section{Conclusión}

El estudio de los instrumentos modernos de gestión, vistos como una manera de conocer a las organizaciones, nos ha permitido dar seguimiento al éxito mundial de algunos de estos instrumentos y a la expansión del papel y el número de consultores.

Desde la mitad de los años ochenta se han desarrollado, en Franciay en algunos otros países en donde está presente nuestra red aca démica, estudios sobre las diversas herramientas de gestión. Estos estudios han demostrado que las investigaciones donde se sigue pa so a paso la vida de estas herramientas permiten comprender muchos aspectos mudos, implícitos, de la vida de las organizaciones. Los instrumentos de gestión son utilizados como métodos muy eficientes para estudiar las estructuras organizativas. Como $\propto$ han implantado en el mundo entero con la misma morfología y la misma fisiología, tienen mucho que enseñarnos en términos de relaciones interculturales. En el futuro nuestra red va a dirigir sus investigaciones en esa dirección. Pero nos parece importante subrayar hoy, que este interés en el estudio de los instrumentos de gestión no puede ser separado del carácter propio de sus estructuras y funciones y de la explosión geo-económica de la importancia de los consultores. 


\section{Bibliografía}

Bayart, D. (1995), “Des objets qui solidifient une théorie: I'histoire du controle statistique de fabrication" en Charue-Duboc F. Des savoirs en action, Paris, L'Harmattan.

Cuq, F. et.al. (1998), "Physiologie et morphologie des dispositifs de gestion", Utinam, no. 4.

Champy, J. (1995), Reengenering M anagement, the Mandate for new leaders hip, New York, Harper Collins.

Dalton, M. (1950), "Conflicts between staff and line managerial officers" American Sociological Review, vol. 15, no. 3, pp. 342-351.

Gabor, A.(1992), The man who discovered quality, Harmonsworth, Penguin.

Ishikwa, K. ( 1984), N ohonteki Hinshitsu Kanri, traducción francesa: Le TQC ou la qualité la japonaise, Paris, AFNOR.

Selznick, Ph. (1949), Tenessee Valley Authority and the Grass Roots, UP, California. 
'òl \%oßI , <"fàl. 
REGIÓN Y SOCIEDAD / VOL. IX / NO. 16. 1998

‘òl \%॰10!1 Y’ú0”-†p@ 